\title{
Integrated triple-collinear counter-terms for the nested soft-collinear subtraction scheme
}

\section{Maximilian Delto and Kirill Melnikov}

Institut für Theoretische Teilchenphysik, Karlsruhe Institute of Technology (KIT), Wolfgang-Gaede Straße 1, Karlsruhe 76128, Germany

E-mail: maximilian.delto@kit.edu, kirill.melnikov@kit.edu

ABSTRACT: We obtain analytic results for integrated triple-collinear splitting functions that emerge as collinear counter-terms in the context of the nested soft-collinear subtraction scheme [1]. With these results, all integrated subtraction terms required for NNLO QCD computations within this scheme are known analytically. In addition to improving efficiency and numerical stability of practical computations, the availability of these results will contribute towards establishing a general NNLO QCD subtraction formula for generic hard scattering processes in hadron collisions, similar to Catani-Seymour and FKS subtractions at NLO.

KEYWORDS: QCD Phenomenology

ARXIV EPRINT: 1901.05213 


\section{Contents}

1 Introduction 1

2 An overview of the nested subtraction scheme and its modification 2

3 The setup 5

$\begin{array}{lll}3.1 & \text { Splittings with a double-soft singularity } & 6\end{array}$

$\begin{array}{lll}3.2 & \text { Triple-collinear splittings without double-soft singularity } & 8\end{array}$

$\begin{array}{lll}4 & \text { Integration of triple-collinear splitting functions } & 9\end{array}$

5 Integration of the strongly-ordered angular limits of triple-collinear split$\begin{array}{lr}\text { ting functions } & 12\end{array}$

$\begin{array}{lll}6 & \text { Extension to the final state radiation } & 12\end{array}$

7 Results for integrated triple-collinear subtraction terms $\quad 14$

$\begin{array}{ll}7.1 \text { Initial state radiation } & 15\end{array}$

$\begin{array}{lll}7.2 & \text { Final state radiation } & 18\end{array}$

8 Conclusion $\quad 19$

\section{Introduction}

An understanding of hard processes that occur in hadron collisions directly from first principles should allow particle physicists to turn the Large Hadron Collider (LHC) into a precision machine and to exploit the huge dataset collected there to search for subtle manifestations of physics beyond the Standard Model. Perturbative QCD plays an important role in this endeavor since it allows for a reliable, systematically improvable description of processes with large momentum transfer. The development of computational techniques for virtual loop amplitudes [2-7] and an improved understanding of how to handle infrared and collinear singularities in real emission processes [8-22, 24, 25] resulted in a large number of theoretical predictions for interesting hard scattering partonic processes at the LHC, accurate through next-to-next-to-leading order [17, 26-46]. It is theoretically interesting and phenomenologically well-motivated to extend such computations to more complex processes; this will require significant improvements in the technology of two-loop computations and the development of more efficient and transparent subtraction schemes.

The nested soft-collinear subtraction scheme introduced in ref. [1] is an attempt to simplify and streamline the sector-improved residue subtraction scheme proposed in refs. [2123]. The key observations made in ref. [1] is that a significant simplification of the subtraction terms can be achieved if subtractions are applied to gauge-invariant scattering 
amplitudes rather than to individual Feynman diagrams and if the subtraction of soft singularities is performed first.

Among the different subtraction terms that are required to make the double-real emission contribution finite, two genuine fully-unresolved contributions - the double-soft and the triple-collinear — stand out, since their analytic computation requires integration over the full phase-space of two unresolved partons. We have recently shown [47] how the doublesoft eikonal function which is sensitive to momenta and color charges of all hard particles that appear in a scattering process can be analytically integrated over the phase-space of the unresolved partons.

The second double-unresolved contribution involves triple-collinear splittings that arise when an initial state parton emits two collinear partons before entering the hard process or a final state parton splits into three collinear partons. These contributions are insensitive to the global structure of any process and can be calculated for each of the external legs separately. The integration of these triple-collinear counter-terms had to be done numerically in ref. [1]. Although, for practical purposes, the need to compute the subtraction terms numerically is hardly a serious problem, it is nevertheless interesting to compute these contributions analytically. Indeed, such a result should allow to check for an analytic cancellation of the infra-red and collinear divergences in a generic hard scattering process and to, hopefully, simplify the finite remainders of the integrated subtraction terms. The analytic integration of the triple-collinear subtraction terms that appear in the context of the nested soft-collinear subtraction scheme [1] is the goal of this paper.

The remainder of the paper is organized as follows. In section 2 we provide a brief overview of the nested soft-collinear subtraction scheme and discuss a small modification of the procedure described in ref. [1] that simplifies an analytic integration of the triple-collinear subtraction terms. In section 3 we precisely define the triple-collinear subtraction terms for the initial state radiation and the phase-space over which they have to be integrated. In section 4 we explain how the phase-space integrals are computed using integration-by-parts identities and differential equations. In section 5 we discuss how the strongly-ordered collinear contributions, required for the subtraction of sub-divergences from the triple-collinear splitting functions, are computed. In section 6 we argue that our calculation can be easily extended to cover the case of the collinear splittings in the final state. In section 7 we present the results for integrated triple-collinear subtraction terms. We conclude in section 8 .

\section{An overview of the nested subtraction scheme and its modification}

The calculation of NNLO QCD corrections to any scattering process requires three ingredients - two-loop virtual corrections, one-loop virtual corrections to a process with an additional final-state parton and the double-real emission contribution. For the purpose of this paper, we are only concerned with the latter. We consider the double-real emission contribution to the production of a final state $X$ in the collision of two partons, i.e. a partonic process $f_{1}+f_{2} \rightarrow X+f_{4}+f_{5}$. Here, $X$ is the "hard" component of the final state, $f_{1,2}$ are the incoming partons and $f_{4,5}$ are the two partons that can become unresolved. 
Although our discussion is applicable in general, we will assume throughout this paper that $X$ is either a vector boson or a Higgs boson. We will assume, for definiteness, that $f_{4,5}$ are gluons in this section but we will present the results for arbitrary integrated triple-collinear splittings later on.

The contribution of the double-real emission process to the cross section reads

$$
\begin{aligned}
\mathrm{d} \sigma_{R R} & =N \int\left[\mathrm{d} g_{4}\right]\left[\mathrm{d} g_{5}\right] \theta\left(E_{4}-E_{5}\right) \mathrm{dLips}_{X}|\mathcal{M}(1,2 ; 4,5 ; X)|^{2} \\
& =\left\langle\left[\mathrm{d} g_{4}\right]\left[\mathrm{d} g_{5}\right] F_{\mathrm{LM}}(1,2 ; 4,5 ; X)\right\rangle .
\end{aligned}
$$

Here, $N$ is a normalization factor and $\operatorname{Lips}_{X}$ is the Lorentz-invariant phase-space of the final state $X$ including the energy-momentum conserving $\delta$-function. The integration measure for each of the final state particles is standard except that an upper energy cut-off is introduced

$$
\left[\mathrm{d} g_{i}\right]=\frac{\mathrm{d}^{d-1} p_{i}}{(2 \pi)^{d-1} 2 E_{i}} \theta\left(E_{\max }-E_{i}\right), \quad i=4,5 .
$$

Since such a cut-off is not Lorentz-invariant, eq. (2.1) should be understood in the centerof-mass frame of the colliding partons. We also note that the gluons are ordered in energy and that $g_{4}$ is always harder than $g_{5}$.

The subtraction terms are constructed iteratively, starting from the so-called doublesoft limit. The double-soft limit is defined as the limit where both $E_{4}$ and $E_{5}$ become vanishingly small but the ratio $E_{5} / E_{4}$ is fixed. We introduce the operator $S$ that extracts the double-soft limit of the differential cross section and write

$$
\begin{aligned}
\left\langle\left[\mathrm{d} g_{4}\right]\left[\mathrm{d} g_{5}\right] F_{\mathrm{LM}}(1,2 ; 4,5 ; X)\right\rangle= & \left\langle\left[\mathrm{d} g_{4}\right]\left[\mathrm{d} g_{5}\right](I-S S) F_{\mathrm{LM}}(1,2 ; 4,5 ; X)\right\rangle \\
& +\left\langle\left[\mathrm{d} g_{4}\right]\left[\mathrm{d} g_{5}\right] S S F_{\mathrm{LM}}(1,2 ; 4,5 ; X)\right\rangle .
\end{aligned}
$$

The first term on the right hand side does not have the double-soft singularity anymore whereas in the second term the action of the double-soft operator causes the two gluons $g_{4,5}$ to decouple from the hard matrix element and the energy-momentum $\delta$-function, so that integration over their momenta can be performed. We obtain

$$
\left\langle\left[\mathrm{d} g_{4}\right]\left[\mathrm{d} g_{5}\right] S S F_{\mathrm{LM}}(1,2 ; 4,5 ; X)\right\rangle=\left\langle F_{\mathrm{LM}}(1,2 ; X)\right\rangle \int\left[\mathrm{d} g_{4}\right]\left[\mathrm{d} g_{5}\right] \operatorname{Eik}(1,2 ; 4,5),
$$

where the double-soft eikonal function is defined through the factorization formula for the matrix element squared

$$
\lim _{E_{4,5} \rightarrow 0}|\mathcal{M}(1,2 ; 4,5 ; X)|^{2}=\operatorname{Eik}(1,2 ; 4,5)|\mathcal{M}(1,2 ; X)|^{2} .
$$

The integral of the double-soft eikonal function in eq. (2.4) was analytically computed in ref. [47] for all relevant hard emittors and unresolved partons.

The term without the double-soft singularity in eq. (2.3) still contains the single-soft singularity that arises in the limit $E_{5} \rightarrow 0$ with $E_{4}$ fixed, and various types of collinear singularities. While the $E_{5} \rightarrow 0$ singularity is treated in exactly the same way as the double-soft ones, subtracting the collinear singularities requires phase-space partitioning. 
The goal of this partitioning is to ensure that only one type of collinear singularity appears in a given sector. This step is described in detail in ref. [1] and we do not repeat it here. In this paper, we focus exclusively on the triple-collinear contributions For example, in case of the triple-collinear emission off the incoming parton $f_{1}$, such a contribution reads

$$
\left\langle\mathbb{C}_{1}\left(I-\sum_{k=a \ldots d} \theta^{k} C_{i_{k} j_{k}}\right)\left[\mathrm{d} g_{4}\right]\left[\mathrm{d} g_{5}\right]\left(I-S_{5}\right)\left(I-S_{S}\right) F_{\mathrm{LM}}(1,2 ; X ; 4,5)\right\rangle .
$$

In the above formula, $\theta_{k \in a, b, c, d}$ stands for partitioning functions that define the individual collinear sectors, which satisfy

$$
\sum_{k=a \ldots d} \theta_{k}=1
$$

Moreover, $\mathbb{C}_{1}$ denotes the triple-collinear limit, where both emitted particles $g_{4,5}$ become collinear to the incoming parton $f_{1}$ but there is no hierarchy of angles between momenta $\vec{p}_{1}, \vec{p}_{4}$ and $\vec{p}_{5}$. In ref. [1] the triple-collinear limit was defined in the context of the parametrization introduced in ref. [22]. In particular, the left-most identity operator was written as a sum of four sectors

$$
\left(I-\sum_{k=a \ldots d} \theta^{k} C_{i_{k} j_{k}}\right) \rightarrow \sum_{k=a \ldots d} \theta^{k}\left(I-C_{i_{k} j_{k}}\right) .
$$

In each of these sectors the relevant scalar products are parametrized by two variables $x_{3}$ and $x_{4}$. The variable $x_{3}$ controls the relative angles between the hard emittor (say parton 1) and the two gluons 4 and 5 , so that $x_{3} \rightarrow 0$ limit corresponds to the triple-collinear limit of the matrix element squared and the phase-space of the two gluons. ${ }^{1}$ Alternatively, we can define the triple-collinear operator that appears in eq. (2.6) together with eq. (2.8) as the $x_{3} \rightarrow 0$ in the parameterization that is used in each of the four sectors [1]. On the contrary, the other parameter, $x_{4}$, controls collinear sub-divergences that arise in different sectors (see ref. [1] for details). These sub-divergences also occur for $x_{3} \neq 0$ and are removed by $C_{i_{k} j_{k}}$ operators that appear in eq. (2.6).

The framework described above was implemented in ref. [1] where it was shown how eq. (2.6) can be integrated numerically. As we already explained, the key element of the approach described in ref. [1] was the identification of the action of the triple-collinear operators $\mathbb{C}_{1}$ and $\mathbb{C}_{2}$ with the extraction of the leading singularity that arises in the $x_{3} \rightarrow 0$ limit. This $x_{3} \rightarrow 0$ limit was taken in the matrix element, in the resolved and un-resolved phase-spaces and in the triple-collinear splitting function. Although formally correct and practically stable, this procedure impacts properties of the unresolved phase space in a way that makes further analytic integrations very difficult.

To overcome this problem, it is useful to re-define the triple-collinear operators $\mathbb{C}_{1,2}$, making them independent of the sector parametrization. Indeed, suppose we postulate that the $\mathbb{C}_{1,2}$ operators act exclusively on the matrix element squared and on the energymomentum conserving delta-function and that they produce products of the triple-collinear

\footnotetext{
${ }^{1}$ We remind the reader that the operators are supposed to act on anything that appears to the right of them including e.g. the phase-space of the unresolved gluons.
} 
splitting functions [49] and reduced matrix elements squared. Considering collinear emissions off parton $f_{1}$ for the sake of example, we define

$$
\mathbb{C}_{1} F_{L M}(1,2,4,5, X)=\frac{g_{s}^{4}}{s_{145}^{2}} P_{f_{1} g_{4} g_{5}}\left(-s_{14},-s_{15}, s_{45}\right) F_{L M}\left(\frac{E_{1}-E_{4}-E_{5}}{E_{1}} \cdot 1,2 ; X\right) .
$$

We emphasize that in this formulation the triple-collinear operator does not act on the unresolved phase-space and does not simplify the scalar products of four-momenta that appear as arguments of the triple-collinear splitting function.

It is easy to see that this reformulation leads to significant simplifications. Indeed, in this case, the triple-collinear subtraction term is integrated over the full unresolved phase-space of two gluons with additional constraint on the sum of their energies. The advantage in this procedure is that the integrand remains a rotationally invariant function in $d-1$ spatial dimensions. We will show below that such integrals can be mapped onto loop integrals and computed in a straightforward way using the method of reverse unitarity [48]. We also note that the strongly-ordered subtraction terms of the form $\theta^{k} C_{i_{k} j_{k}}$ are computed sector by sector and rely on a particular choice of the phase-space parametrization. It turns out, however, that these strongly-ordered subtraction terms simplify sufficiently to allow for a straightforward analytic integration in terms of Gamma-functions.

Thus, a re-definition of a triple-collinear operator, compared to the original proposal in ref. [1], allows us to complete the analytic integration of the double-unresolved subtraction terms that arise within the framework of the nested soft-collinear subtraction scheme. In the remaining sections of this paper, we will describe our computation in detail and present the results for the integrated collinear counter-terms for a variety of triple-collinear splitting functions.

\section{The setup}

In this section, we precisely define the triple-collinear counter-terms that need to be integrated. As we explain below, it is convenient to use two different energy parametrizations for the emitted partons. We generalize eq. (2.6) to the case of arbitrary partons and assume that the triple-collinear operator is defined as explained in the previous section. We obtain

$$
\begin{aligned}
\mathcal{I}_{\mathrm{TC}}= & \left\langle\mathbb{C}_{1}\left(I-\sum_{k=a \ldots d} \theta^{k} C_{i_{k} j_{k}}\right)\left(I-S_{5}\right)\left(I-S_{S}\right) F_{\mathrm{LM}}(1,2 ; X ; 4,5)\right\rangle \\
= & g_{s}^{4}\left\langle\left(I-\sum_{k=a \ldots d} \theta^{k} C_{i_{k} j_{k}}\right)\left(I-S_{5}\right)(I-\mathscr{S})\left[\mathrm{d} f_{4}\right]\left[\mathrm{d} f_{5}\right] \frac{P_{f_{1} f_{4} f_{5}}\left(-s_{14},-s_{15}, s_{45}, z_{4}, z_{5}\right)}{s_{145}^{2}}\right. \\
& \left.\times F_{\mathrm{LM}}\left(\left(1-\frac{E_{4}}{E_{1}}-\frac{E_{5}}{E_{1}}\right) \cdot 1,2\right)\right\rangle .
\end{aligned}
$$

All necessary triple-collinear splitting functions are computed in e.g. ref. [49]. ${ }^{2}$ We note that the minus signs in front of some of its arguments appear because we consider collinear

\footnotetext{
${ }^{2}$ We stress that we only need spin-averaged splitting functions in the triple-collinear limits [50].
} 
radiation off an initial state parton. For the same reason, the energy fractions $z_{4,5}$ and the off-shellness of the hard parton $s_{145}$ read

$$
z_{4,5}=E_{4,5} /\left(E_{4}+E_{5}-E_{1}\right), \quad s_{145}=-s_{14}-s_{15}+s_{45} .
$$

It follows from eq. (3.1) that the hard matrix element depends on the sum over energies of the emitted partons; hence, it is possible to integrate over angles of the final state partons $f_{4,5}$ and their energy fractions, keeping the sum of their energies fixed. It turns out to be convenient to define these energy fractions differently depending on whether or not a particular final state has a double-soft singularity. The two different parametrizations are discussed in sections 3.1 and 3.2 , respectively.

\subsection{Splittings with a double-soft singularity}

We begin with the discussion of the energy parametrization that is mostly used for processes that exhibit double-soft singularities; the prominent examples include $g \rightarrow g^{*}+g g$ and $q \rightarrow q^{*}+g g$ initial state splittings. The variable transformations that are used in this case are described in ref. [1]; for the sake of completeness we repeat this discussion here. To this end, we re-write eq. (3.1) as

$$
\mathcal{I}_{\mathrm{TC}}=\left\langle\left(I-S_{5}\right)(I-S S) \mathrm{d} T_{C}\left(s_{14}, s_{15}, s_{45}, E_{4}, E_{5}\right) F_{\mathrm{LM}}\left(E_{145} / E_{1} \times 1,2\right)\right\rangle,
$$

where $E_{145}=E_{1}-E_{4}-E_{5}$ and

$$
\mathrm{d} T_{C}=\left(I-\sum_{k=a \ldots d} \theta_{k} C_{k}\right)\left[\mathrm{d} g_{4}\right]\left[\mathrm{d} g_{5}\right] \frac{g_{s}^{4}}{s_{145}^{2}} P_{f_{1} f_{4} f_{5}}\left(-s_{14},-s_{15}, s_{45}, z_{4}, z_{5}\right) .
$$

Isolating integrations over energies we obtain

$$
\mathrm{d} T_{C}=\mathrm{d} E_{4} \mathrm{~d} E_{5} E_{4}^{1-2 \epsilon} E_{5}^{1-2 \epsilon} \theta\left(E_{4}-E_{5}\right) \theta\left(E_{\max }-E_{4}\right) \widetilde{T}_{C}\left(E_{4}, E_{5}, E_{1}\right)
$$

where

$$
\widetilde{T}_{C}\left(E_{4}, E_{5}, E_{1}\right)=\int\left(I-\sum_{k=a \ldots d} \theta_{k} C_{k}\right) \mathrm{d} \Omega_{45} \frac{g_{s}^{4}}{s_{145}^{2}} P_{f_{1} f_{4} f_{5}}\left(-s_{14},-s_{15}, s_{45}, z_{4}, z_{5}\right),
$$

where $\Omega_{45}=\frac{\mathrm{d} \Omega_{4}^{(d-1)} \mathrm{d} \Omega_{5}^{(d-1)}}{2^{2}(2 \pi)^{2 d-2}}$ is the angular integration measure. We use these expressions in eq. (3.3) and derive

$$
\mathcal{I}_{\mathrm{TC}}=\int_{E_{4}>E_{5}}\left(I-S_{S}\right)\left(I-S_{5}\right) \mathrm{d} E_{4} \mathrm{~d} E_{5}\left(E_{4} E_{5}\right)^{1-2 \epsilon} \widetilde{T}_{C}\left(E_{4}, E_{5}, E_{1}\right) F_{\mathrm{LM}}\left(\frac{E_{145}}{E_{1}} \cdot 1,2\right) .
$$

To proceed further we need to choose an energy parametrization that decouples hard matrix element from the splitting function. In this section, we consider the parametrization discussed in ref. [1]. For the hard collinear emission we use

$$
E_{4}=E_{1}(1-z)(1-r / 2), \quad E_{5}=E_{1}(1-z) r / 2
$$


with $0<z<1$ and $0<r<1$. This parametrization automatically satisfies the constraint $E_{4}>E_{5}$ and makes hard scattering matrix element $r$-independent

$$
\left\langle F_{\mathrm{LM}}\left(E_{145} / E_{1} \cdot 1,2\right)\right\rangle=\left\langle F_{\mathrm{LM}}(z \cdot 1,2)\right\rangle .
$$

However, this parametrization is not optimal for the soft subtraction. Indeed, singlesoft subtraction terms that are obtained when operator $S_{5}$ acts on matrix elements are computed by taking the limit $E_{5} \rightarrow 0$ at fixed $E_{4}$ [1]. The parametrization in eq. (3.8) does not allow us to easily do that; for this reason, we have to switch to a different parametrization to describe the single-soft limit. It is convenient to choose

$$
E_{4}=E_{1}(1-z), \quad E_{5}=E_{1}(1-z) r .
$$

After some manipulations described in ref. [1], integrated triple-collinear subtraction terms can be cast into the following form

$$
\mathcal{I}_{\mathrm{TC}}=\left[\alpha_{s}\right]^{2} E_{1}^{-4 \epsilon} \int_{0}^{1} \mathrm{~d} z\left[R_{\delta} \delta(1-z)+\frac{R_{+}}{\left[(1-z)^{1+4 \epsilon}\right]_{+}}+R_{\mathrm{reg}}(z)\right]\left\langle\frac{F_{\mathrm{LM}}(z \cdot 1,2)}{z}\right\rangle,
$$

where $\left[\alpha_{s}\right]=\left[\frac{\alpha_{s}(\mu) \mu^{2 \epsilon} e^{\epsilon \gamma_{E}}}{2 \pi \Gamma(1-\epsilon)}\right]$ is a parameter related to the strong coupling constant,

$$
\frac{1}{\left[(1-z)^{1+4 \epsilon}\right]_{+}}=\left[\frac{1}{1-z}\right]_{+}-4 \epsilon\left[\frac{\log (1-z)}{1-z}\right]_{+}+\ldots
$$

and $R_{\delta,+, \text { reg }}$ read

$$
\begin{aligned}
R_{\delta} & =\frac{\left(E_{\max } / E_{1}\right)^{-4 \epsilon}-1}{4 \epsilon} A_{3}-\int_{0}^{1} \frac{\mathrm{d} r}{r^{1+2 \epsilon}} \frac{\left[(1+r)^{4 \epsilon}-1\right]}{4 \epsilon} F(r), \\
R_{+} & =A_{1}(1)+A_{2}(1), \\
R_{\mathrm{reg}}(z) & =\frac{A_{1}(z)+A_{2}(z)-A_{1}(1)-A_{2}(1)}{(1-z)^{1+4 \epsilon}} .
\end{aligned}
$$

To write down expressions for one constant $A_{1}$ and three functions $A_{2,3}$ and $F$ it is convenient to introduce a new operator that, acting on a function, extracts its limit when a particular variable is set to zero

$$
\mathcal{T}_{x} g(\ldots, x, \ldots)=\lim _{x \rightarrow 0} g(., x, \ldots)=g(\ldots, 0 \ldots) .
$$

We find

$$
\begin{aligned}
A_{1}(z)= & \frac{z(1-z)^{4}}{2^{-2 \epsilon}} \int_{0}^{1} \frac{\mathrm{d} r}{r^{1+2 \epsilon}}\left(1-\frac{r}{2}\right)^{-1-2 \epsilon} \times\left(1-\mathcal{T}_{r}\right)\left[\left(\frac{r}{2}\right)^{2}\left(1-\frac{r}{2}\right)^{2}\right. \\
& \left.\times E_{1}^{4} \widetilde{T}_{C}\left(E_{1}, E_{1}(1-z)\left(1-\frac{r}{2}\right), E_{1}(1-z) \frac{r}{2}\right)\right]
\end{aligned}
$$


and

$$
\begin{aligned}
A_{2}(z) & =\frac{z(1-z)^{4}}{2 \epsilon}\left[1-\frac{\Gamma^{2}(1-2 \epsilon)}{\Gamma(1-4 \epsilon)}\right] \mathcal{T}_{r}\left[r^{2} E_{1}^{4} \widetilde{T}_{C}\left(E_{1}, E_{1}(1-z), E_{1}(1-z) r\right)\right], \\
A_{3} & =\int_{0}^{1} \frac{\mathrm{d} r}{r^{1+2 \epsilon}} \mathcal{T}_{1-z}\left[(1-z)^{4}\left(1-\mathcal{T}_{r}\right)\left[r^{2} E_{1}^{4} \widetilde{T}_{C}\left(E_{1}, E_{1}(1-z), E_{1}(1-z) r\right)\right]\right], \\
F(r) & =\mathcal{T}_{1-z}\left[(1-z)^{4} r^{2} E_{1}^{4} \widetilde{T}_{C}\left(E_{1}, E_{1}(1-z), E_{1}(1-z) r\right)\right] .
\end{aligned}
$$

We will explain how to compute these functions in the following section.

\subsection{Triple-collinear splittings without double-soft singularity}

Several cases of triple-collinear splittings do not exhibit the double-soft singularities and where only one of the final state partons may cause a single-soft singularity. A typical example is the splitting $g \rightarrow q^{*}+q g$. In this case, we do not introduce the energy ordering and parametrize the two energies as follows

$$
E_{4}=E_{1}(1-z)(1-r), \quad E_{5}=E_{1}(1-z) r .
$$

We note that the parametrization of energies in eq. (3.17) is chosen in such a way that $r=0$ corresponds to the single-soft limit; this is then reflected in how $E_{4,5}$ are assigned to final state partons. For example, in case of $g \rightarrow q^{*}+q g$ splitting, we choose $E_{5}$ to be the energy of the final state gluon and $E_{4}$ to be the energy of the final state on-shell quark.

Following steps similar to those described in the previous section, we write the integrated triple-collinear subtraction term in the following way

$$
\mathcal{I}_{\mathrm{TC}}=\left[\alpha_{s}\right]^{2} E_{1}^{-4 \epsilon} \int_{0}^{1} \mathrm{~d} z \tilde{R}_{\mathrm{reg}}(z)\left\langle\frac{F_{\mathrm{LM}}(z \cdot 1,2)}{z}\right\rangle,
$$

where

$$
\tilde{R}_{\mathrm{reg}}(z)=z(1-z)^{3-4 \epsilon}\left[\tilde{A}_{1}(z)+\tilde{A}_{2}(z)\right] .
$$

The two functions that appear in eq. (3.19) are defined as follows

$$
\begin{aligned}
& \tilde{A}_{1}(z)=\int_{0}^{1} \frac{\mathrm{d} r}{r^{1+2 \epsilon}}(1-r)^{1-2 \epsilon}\left(1-\mathcal{T}_{r}\right)\left[r^{2} E_{1}^{4} \widetilde{T}_{C}\left(E_{1}, E_{1}(1-z)(1-r), E_{1}(1-z) r\right)\right], \\
& \tilde{A}_{2}(z)=\frac{1}{2 \epsilon}\left[\left(E_{\max } / E_{1}\right)^{-2 \epsilon}(1-z)^{2 \epsilon}-\frac{(1-2 \epsilon)}{(1-4 \epsilon)} \frac{\Gamma^{2}(1-2 \epsilon)}{\Gamma(1-4 \epsilon)}\right] \mathcal{T}_{r}\left[r^{2} E_{1}^{4} \widetilde{T}_{C}\left(E_{1}, E_{1}(1-z), E_{1}(1-z) r\right)\right] .
\end{aligned}
$$

Their calculation is described in the following section. 


\section{Integration of triple-collinear splitting functions}

Our goal is the analytic computation of $R_{\delta}, R_{+}$and $R_{\text {reg }}(z)$ or $\tilde{R}_{\text {reg }}(z)$ for all the different splitting functions. There are two ingredients required for such a computation, as can be seen by inspecting eq. (3.6). One of them is the integral of the triple-collinear splitting function over the angles of unresolved gluons. The second ingredient is the integral of the strongly-order collinear subtraction terms. These terms are particular for each of the four sectors that appear in eq. (2.6). Both of these contributions need to be further integrated over $r$, as discussed in the previous section.

We begin with the integration of the triple-collinear splitting function over the phasespace of unresolved partons. We consider the following integral

$$
W_{a b c}\left(E_{1}, E_{4}, E_{5}\right)=\int \mathrm{d} \Omega_{45} P_{a b c}\left(-s_{14},-s_{15}, s_{45}, z_{4}, z_{5}\right) / s_{145}^{2},
$$

where $P_{a b c}$ is a triple-collinear splitting function. It is essential that the integration over angles in eq. (4.1) is not restricted to the collinear region; we emphasized this point earlier and this is an important modification of the subtraction scheme described in ref. [1].

The resulting integral $W_{a b c}$ is a function of energies of the relevant partons. To compute it, we employ methods of multi-loop computations such as integration-by-parts and differential equations. The connection between loop and phase-space integrals is provided by reverse unitarity [48]. To re-write the integral $W_{a b c}$ in a way that allows one to apply reverse unitarity, we re-introduce integration over three-momenta of partons 4 and 5 . We obtain

$$
W_{a b c}=\int\left[\mathrm{d} g_{4}\right]\left[\mathrm{d} g_{5}\right] \frac{\delta\left(k_{4}^{0}-E_{4}\right) \delta\left(k_{5}^{0}-E_{5}\right)}{\left(E_{4} E_{5}\right)^{1-2 \epsilon} s_{145}^{2}} P_{a b c}\left(-s_{14},-s_{15}, s_{45}, z_{4}, z_{5}\right) .
$$

We write partonic energies $k_{i}^{0}$ in the covariant form by introducing auxiliary vector $P=$ $(1 / 2, \overrightarrow{0})$; then $\delta\left(k_{i}^{0}-E_{i}\right)=\delta\left(2 k_{i} \cdot P-E_{i}\right)$. Since $\left[\mathrm{d} g_{i}\right]=\mathrm{d}^{d} k_{i} \delta_{+}\left(k_{i}^{2}\right)$, we can easily turn a phase-space integral in eq. (4.2) into a loop integral by replacing all $\delta$-functions with the corresponding "propagators"; we will refer to these propagators as "cut". Hence, to compute $W_{a b c}$, we need to consider the following class of loop integrals

$$
I_{a_{5}, a_{6}, a_{7}, a_{8}}\left(E_{1}, E_{4}, E_{5}\right)=\int \frac{\mathrm{d}^{d} k_{4}}{(2 \pi)^{d}} \frac{\mathrm{d}^{d} k_{5}}{(2 \pi)^{d}} \frac{1}{D_{1} D_{2} D_{3} D_{4} D_{5}^{a_{5}} D_{6}^{a_{6}} D_{7}^{a_{7}} D_{8}^{a_{8}}},
$$

where

$$
\begin{array}{llll}
D_{1}=k_{4}^{2}, & D_{2}=k_{5}^{2}, & D_{3}=2 P \cdot k_{4}-E_{4}, & D_{4}=2 P \cdot k_{5}-E_{5}, \\
D_{5}=\left(p_{1}+k_{4}\right)^{2}, & D_{6}=\left(p_{1}+k_{5}\right)^{2}, & D_{7}=\left(k_{4}+k_{5}\right)^{2}, & D_{8}=\left(p_{1}+k_{4}+k_{5}\right)^{2} .
\end{array}
$$

We note that propagators $1 / D_{1, \ldots, 4}$ need to be "cut", in reverse unitarity sense, so that we will only consider integrals where these propagators are raised to first power.

The importance of mapping phase-space integrals onto two-loop integrals stems from the fact that we can use well-established methods to find algebraic relations between phasespace integrals that follow from integration-by-parts identities. With these relations at 
hand, we can identify a minimal set of integrals, the so-called master integrals, that have to be computed, to obtain the function $W_{a b c}$.

The master integrals satisfy first order differential equations in kinematic variables; solving them as an expansion in the dimensional regulator $\epsilon=(4-d) / 2$ yields the required integrals up to boundary constants that have to be obtained using different methods. We have used the computer program Reduze2 [51] to identify master integrals and to derive the differential equations. We note that there exist linear relations between inverse propagators $D_{1,2}, D_{5,6,7,8}$ that are used to reduce the number of independent integrals.

For our calculation, we choose the following set of independent master integrals

$$
\boldsymbol{I}=\left\{I_{0,0,0,0}, I_{0,0,0,1}, I_{-1,0,0,2}, I_{0,-1,0,2}\right\}
$$

To derive the differential equations, it is convenient to introduce dimensionless variables

$$
E_{i} \rightarrow \omega_{i}=\frac{E_{i}}{E_{1}}, \quad i=4,5,
$$

and study integrals $I_{a_{4}, a_{5}, a_{6}, a_{7}}$ as functions of $E_{1}$ and $\omega_{4,5}$. The dependence of these integrals on $E_{1}$ follows from their mass dimensions. Hence, we define

$$
I_{a_{5}, a_{6}, a_{7}, a_{8}}\left(E_{1}, E_{4}, E_{5}\right)=E_{1}^{2 d-6-2\left(a_{5}+a_{6}+a_{7}+a_{8}\right)} \bar{I}_{a_{5}, a_{6}, a_{7}, a_{8}}\left(\omega_{4}, \omega_{5}\right)
$$

and study the dependence of the integrals $\bar{I}$ on $\omega_{4,5}$.

Using integration-by-parts identities, it is straightforward to derive first-order differential equations for master integrals. It is, however, well-known that it is beneficial to transform a system of equations to a canonical form [52]. We achieve this by applying the algorithmic approach of ref. [53] sequentially in both variables. We find

$$
\mathrm{d} \overline{\boldsymbol{J}}=\frac{\epsilon}{20} \sum_{i=4,5} \mathrm{~d} \hat{M}_{\omega_{i}}\left(\omega_{4}, \omega_{5}, \epsilon\right) \times \overline{\boldsymbol{J}}
$$

The linear relation between elements of the canonical basis $\overline{\boldsymbol{J}}$ and the original master integrals $\overline{\boldsymbol{I}}$

$$
\overline{\boldsymbol{I}}=\hat{T} \overline{\boldsymbol{J}}
$$

is specified by the transformation matrix

$$
\hat{T}=\left(\begin{array}{cccc}
\omega_{4} \omega_{5} & 0 & 0 & 0 \\
0 & \frac{(1-2 \epsilon)^{2}\left(2 \omega_{4}+2 \omega_{5}-1\right)}{\epsilon(1-6 \epsilon)} & -\frac{(1-2 \epsilon)^{2}\left(\omega_{4}+1\right)}{\epsilon(1-6 \epsilon)} & -\frac{(1-2 \epsilon)^{2}\left(\omega_{5}+1\right)}{\epsilon(1-6 \epsilon)} \\
0 & -\frac{2(1-2 \epsilon)^{2}\left(4 \omega_{4} \epsilon-2 \omega_{5} \epsilon+\epsilon-\omega_{4}\right)}{\epsilon(1-6 \epsilon)} & \frac{(1-2 \epsilon)^{2}\left(4 \omega_{4} \epsilon-2 \epsilon-\omega_{4}\right)}{\epsilon(1-6 \epsilon)} & -\frac{2(1-2 \epsilon)^{2}\left(\omega_{5}+1\right)}{(1-6 \epsilon)} \\
0 & \frac{2(1-2 \epsilon)^{2}\left(2 \omega_{4} \epsilon-4 \omega_{5} \epsilon-\epsilon+\omega_{5}\right)}{\epsilon(1-6 \epsilon)} & -\frac{2(1-2 \epsilon)^{2}\left(\omega_{4}+1\right)}{(1-6 \epsilon)} & \frac{(1-2 \epsilon)^{2}\left(4 \omega_{5} \epsilon-2 \epsilon-\omega_{5}\right)}{\epsilon(1-6 \epsilon)}
\end{array}\right)
$$


The two matrices that appear in the canonical differential equation eq. (4.8) read

$$
\begin{aligned}
& \mathrm{d} \hat{M}_{\omega_{4}}=\mathrm{d} \ln \left(\omega_{4}\right) \times\left(\begin{array}{cccc}
-40 & 0 & 0 & 0 \\
1 & -12 & 16 & 0 \\
-3 & 36 & -48 & 0 \\
2 & -24 & 32 & 0
\end{array}\right)+\mathrm{d} \ln \left(\omega_{4}-1\right) \times\left(\begin{array}{cccc}
0 & 0 & 0 & 0 \\
-2 & -8 & 0 & -16 \\
1 & 4 & 0 & 8 \\
-4 & -16 & 0 & -32
\end{array}\right) \\
& +\operatorname{dln}\left(\omega_{4}+\omega_{5}-1\right) \times\left(\begin{array}{cccc}
0 & 0 & 0 & 0 \\
3 & -60 & 0 & 0 \\
1 & -20 & 0 & 0 \\
1 & -20 & 0 & 0
\end{array}\right)+\operatorname{dln}\left(\omega_{4}+\omega_{5}\right) \times\left(\begin{array}{cccc}
0 & 0 & 0 & 0 \\
-2 & -24 & 16 & 16 \\
1 & 12 & -8 & -8 \\
1 & 12 & -8 & -8
\end{array}\right) \text {, } \\
& \mathrm{d} \hat{M}_{\omega_{5}}=\operatorname{dln}\left(\omega_{5}\right) \times\left(\begin{array}{cccc}
-40 & 0 & 0 & 0 \\
1 & -12 & 0 & 16 \\
2 & -24 & 0 & 32 \\
-3 & 36 & 0 & -48
\end{array}\right)+\mathrm{d} \ln \left(\omega_{5}-1\right) \times\left(\begin{array}{cccc}
0 & 0 & 0 & 0 \\
-2 & -8 & -16 & 0 \\
-4 & -16 & -32 & 0 \\
1 & 4 & 8 & 0
\end{array}\right) \\
& +\operatorname{dln}\left(\omega_{4}+\omega_{5}-1\right) \times\left(\begin{array}{cccc}
0 & 0 & 0 & 0 \\
3 & -60 & 0 & 0 \\
1 & -20 & 0 & 0 \\
1 & -20 & 0 & 0
\end{array}\right)+\operatorname{dln}\left(\omega_{4}+\omega_{5}\right) \times\left(\begin{array}{cccc}
0 & 0 & 0 & 0 \\
-2 & -24 & 16 & 16 \\
1 & 12 & -8 & -8 \\
1 & 12 & -8 & -8
\end{array}\right) \text {. }
\end{aligned}
$$

It is straightforward to integrate this system of differential equations as an expansion in the dimensional regularization parameter $\epsilon$. However, to fully determine the integrals, we require boundary conditions. To this end, we note that one of the master integrals the phase-space - can be straightforwardly computed. It reads

$$
\bar{I}_{0,0,0,0}=\frac{\left(\omega_{4} \omega_{5}\right)^{1-2 \epsilon}}{16}\left[\Omega^{d-1}\right]^{2} .
$$

The boundary conditions for all other integrals are obtained by considering the limit $\omega_{4}=$ $\omega_{5}=\omega \rightarrow 0$. It follows from differential equations that the boundary conditions are obtained from the $\omega^{n-4 \epsilon}$-branches of master integrals, where $n$ is an integer number. We obtain those branches by expanding denominators that appear in master integrals in Taylor series in $\omega$. As an example, we consider the integral $I_{0,0,0,1}$; expanding the denominator in $\omega$, we obtain

$$
I_{0,0,0,1}=I_{0,0,0,0} \omega^{-1} \int \frac{\mathrm{d} \Omega_{45}}{\left[\Omega^{d-1}\right]^{2}} \frac{1}{\left[\eta_{14}+\eta_{15}\right]}+\ldots
$$

where ellipses stand for less singular terms in the $\omega$-expansion. We compute the integral over angles and find

$$
\begin{aligned}
\int \frac{\mathrm{d} \Omega_{45}}{\left[\Omega^{d-1}\right]^{2}} \frac{1}{\left[\eta_{14}+\eta_{15}\right]}= & \frac{(1-2 \epsilon)^{2}}{\epsilon(1-4 \epsilon)} \frac{\Gamma^{4}(1-2 \epsilon) \Gamma(1+\epsilon)}{\Gamma(1-4 \epsilon) \Gamma^{3}(1-\epsilon)} \\
& -\frac{1-2 \epsilon}{\epsilon} \times{ }_{3} F_{2}[\{1,1-\epsilon, 2 \epsilon\},\{2(1-\epsilon), 1+\epsilon\} ;-1] .
\end{aligned}
$$


A simple analysis shows that the boundary conditions for the remaining two integrals are related to the angular integral in eq. (4.15).

The differential equations presented in eq. (4.11) and eq. (4.12), together with the boundary condition eqs. (4.13), (4.15) provide the starting point for the computation of the function $\widetilde{T}_{C}$ for any splitting and in any energy parameterization. For every parameterization, we perform a change of variables in the differential equations and solve them in terms of Goncharov Polylogarithms (GPLs) [54]. The resulting integrals contain GPLs of two types: 1) $G(\{\vec{a}(z)\}, r)$, where vectors $\vec{a}(z)$ is a rational function of $z$ and 2) $G(\{\vec{b}\}, x)$ where $x=r, z$ and with components of $\vec{b}$ being rational numbers. We note that - with master integrals written in this way - the integration over the $r$-variable becomes straightforward. Finally, we numerically checked the master integrals by deriving Mellin-Barnes representations and integrating them using the MB.m package [55] for a few values of $E_{4,5}$.

\section{Integration of the strongly-ordered angular limits of triple-collinear splitting functions}

To complete the computation of the integrated triple-collinear counter-term, we require the strongly-ordered subtraction terms described by the following equation

$$
g_{s}^{4} \sum_{k=a \ldots d}\left\langle\theta_{k} C_{k}\left[\mathrm{~d} f_{4}\right]\left[\mathrm{d} f_{5}\right] \frac{P_{f_{1} f_{4} f_{5}}\left(-s_{14},-s_{15}, s_{45}, z_{4}, z_{5}\right)}{s_{145}^{2}} F_{\mathrm{LM}}\left(\frac{E_{145}}{E_{1}} \cdot 1,2\right)\right\rangle .
$$

This computation proceeds in the following way. For each of the four sectors, we employ the phase-space parametrization of ref. [21]. The relevant formulas for scalar products of four-momenta and for the phase-space in each of the four sectors can be found in the appendix B of ref. [1]. In terms of this parametrization, the strongly-ordered angular limits correspond to $x_{4} \rightarrow 0$ limit which, according to our earlier discussion, needs to be taken at fixed, non-vanishing $x_{3}$. It is easy to check that, upon taking this limit, the scalar products and the unresolved phase-space simplify dramatically so that integration over two remaining variables $x_{3}$ and $\lambda$ [21] can be trivially performed and leads to a rational function of $r$ and $z$ that can be further integrated over $r$ in a straightforward way. We note that both the integral of the triple-collinear splitting function and the integral of its strongly-ordered limit start at $1 / \epsilon^{2}$ which, however, must cancel when the difference of the two is taken. This cancellation provides a welcome consistency check of the calculation.

\section{Extension to the final state radiation}

Up to now we have focused on the initial state radiation. In this section we argue that identical techniques can be used to perform analytic integration of subtraction terms for triple-collinear radiation off a final state parton. For the sake of simplicity, we focus on quark-initiated splittings $q^{*} \rightarrow q_{1}+f_{4}+f_{5}$. In the context of the nested soft-collinear subtraction scheme, these final state integrated collinear counter-terms were discussed in ref. [56] where the NNLO QCD corrections to Higgs decay to $b \bar{b}$ pairs were investigated. Without going into details, we note that for the final state radiation the hard matrix 
element fully decouples. The integrated triple collinear limit is then a function of $\epsilon$ that is obtained by computing the following integral

$$
\begin{aligned}
\mathcal{I}_{\mathrm{TC}}= & E^{-4 \epsilon}\left\{\int_{0}^{1} \frac{\mathrm{d} x_{1}}{x_{1}^{1+4 \epsilon}} \frac{\mathrm{d} x_{2}}{x_{2}^{1+2 \epsilon}}\left(1-\mathcal{T}_{x_{1}}\right)\left(1-\mathcal{T}_{x_{2}}\right)\left(1-x_{1}-x_{1} x_{2}\right)^{n-2 \epsilon} \times\right. \\
& \times \theta\left(1-x_{1}-x_{1} x_{2}\right)\left[E^{4} x_{1}^{4} x_{2}^{2} \widetilde{T}_{C}\left(E\left(1-x_{1}-x_{1} x_{2}\right), E x_{1}, E x_{1} x_{2}\right)\right] \\
& \left.-\frac{\left(E_{\max } / E\right)^{-4 \epsilon}-1}{-4 \epsilon} \int_{0}^{1} \frac{\mathrm{d} x_{2}}{x_{2}^{1+2 \epsilon}} \mathcal{T}_{x_{1}}\left(1-\mathcal{T}_{x_{2}}\right)\left[E^{4} x_{1}^{4} x_{2}^{2} \widetilde{T}_{C}\left(E\left(1-x_{1}-x_{1} x_{2}\right), E x_{1}, E x_{1} x_{2}\right)\right]\right\},
\end{aligned}
$$

where $\widetilde{T}_{C}$ is obtained from a similar quantity defined in section 3 except that we need to change $s_{1 k} \rightarrow-s_{1 k}$ and $E_{1} \rightarrow-E_{1}$ in there. After that replacement, energies are parametrized as follows

$$
E_{4}=E x_{1}, \quad E_{5}=E x_{1} x_{2}, \quad E_{1}=E\left(1-x_{1}-x_{1} x_{2}\right) .
$$

In the integral eq. (6.1), the dependence on $E_{\max }$ is due to the cut-off theta function $\theta\left[E_{\max }-E_{4}\right]=\theta\left[E_{\max } / E-x_{1}\right]=\theta^{E}$, which is, however only relevant for terms that contain the double-soft limit. For all other contributions, the energy conservation condition $\theta\left(1-x_{1}-x_{1} x_{2}\right)$ provides a stronger bound. To arrive at eq. (6.1), we divide the integrand as follows

$$
\begin{aligned}
\theta^{E}\left(1-\mathcal{T}_{x_{1}}\right)\left(1-\mathcal{T}_{x_{2}}\right) & =\left[\left(1-\mathcal{T}_{x_{2}}\right)-\theta^{E} \mathcal{T}_{x_{1}}\left(1-\mathcal{T}_{x_{2}}\right)\right] \\
& =\left[\left(1-\mathcal{T}_{x_{2}}\right)-\mathcal{T}_{x_{1}}\left(1-\mathcal{T}_{x_{2}}\right)\right]-\left(\theta^{E}-1\right) \mathcal{T}_{x_{1}}\left(1-\mathcal{T}_{x_{2}}\right)
\end{aligned}
$$

We note that the second term in eq. (6.1) is generated by the $\theta^{E}-1$ contribution, it vanishes for splittings that do not exhibit a double soft singularity and in cases where one can choose $E_{\max }=E$. This is for example the case for NNLO corrections to $1 \rightarrow 2$ decays. We also point out that a general integer power $n$ appears in the factor $\left(1-x_{1}-x_{1} x_{2}\right)^{n-2 \epsilon}$ in eq. (6.1). This factor reflects possible energy-dependent damping factors in variants of the subtraction scheme. It will be clear from the follow up discussion that these factors do not pose any problem for the integration.

To compute the angular integrals in $\widetilde{T}_{C}$ we follow the steps outlined in section 4 . Upon integration over angles, we write the result in terms of GPLs with an argument $x_{2}$ where appropriate; this makes the subsequent $x_{2}$ integration straightforward except that we have to respect the $\theta$-function in eq. (6.1). We accomplish that by splitting the integral in the following way

$$
\int_{0}^{1} \mathrm{~d} x_{1} \int_{0}^{1} \mathrm{~d} x_{2} \theta\left(1-x_{1}-x_{1} x_{2}\right)=\int_{0}^{1 / 2} \mathrm{~d} x_{1} \int_{0}^{1} \mathrm{~d} x_{2}+\int_{1 / 2}^{1} \mathrm{~d} x_{1} \int_{0}^{\left(1-x_{1}\right) / x_{1}} \mathrm{~d} x_{2} .
$$

The $x_{2}$ integration results in GPLs that contain constants and rational functions of $x_{1}$ both in the letters and in the arguments. Such GPLs can be re-written in terms of GPLs 


\begin{tabular}{|c|c|c|c|}
\hline Splitting & $P_{a b c}$ & EP & Name in the supplementary material \\
\hline$q \rightarrow g g q^{*}$ & $1 / 2\left(P_{g_{4} g_{5} q_{1}}+4 \leftrightarrow 5\right)$ & 1 & ISR $[\mathrm{z}, 1]$ \\
$g \rightarrow g g g^{*}$ & $1 / 2\left(P_{g_{1} g_{4} g_{5}}+4 \leftrightarrow 5\right)$ & 1 & $\operatorname{ISR}[\mathrm{z}, 2]$ \\
$q \rightarrow \bar{q}^{\prime} q^{\prime} q^{*}$ & $P_{\bar{q}_{4}^{\prime} q_{5}^{\prime} q_{1}}+4 \leftrightarrow 5$ & 1 & $\operatorname{ISR}[\mathrm{z}, 3]$ \\
$q \rightarrow q q^{\prime} \bar{q}^{*}$ & $P_{\bar{q}_{1}^{\prime} q_{4}^{\prime} q_{5}}+4 \leftrightarrow 5$ & 1 & $\operatorname{ISR}[\mathrm{z}, 4]$ \\
$q \rightarrow \bar{q} q q^{*}$ & $P_{\bar{q}_{4} q_{5} q_{1}}+1 \leftrightarrow 5$ & 1 & $\operatorname{ISR}[\mathrm{z}, 5]$ \\
$q \rightarrow q q \bar{q}^{*}$ & $1 / 2\left(P_{\bar{q}_{1} q_{4} q_{5}}^{\mathrm{id}}+4 \leftrightarrow 5\right)$ & 1 & $\operatorname{ISR}[\mathrm{z}, 6]$ \\
$g \rightarrow q \bar{q} g^{*}$ & $P_{g_{1} q_{4} \bar{q}_{5}}+4 \leftrightarrow 5$ & 1 & $\operatorname{ISR}[\mathrm{z}, 7]$ \\
$q \rightarrow q g g^{*}$ & $P_{g_{5} q_{1} \bar{q}_{4}}$ & 2 & $\operatorname{ISR}[\mathrm{z}, 8]$ \\
$g \rightarrow q g q *$ & $P_{g_{1} g_{5} q_{4}}$ & 2 & $\operatorname{ISR}[\mathrm{z}, 9]$ \\
\hline
\end{tabular}

Table 1. Overview of integrated triple-collinear subtraction terms for initial state splittings. In the first column we define the splitting, in the second column, we identify the corresponding triplecollinear splitting functions in ref. [49], where we include an additional symmetry factor where required. In the third column, we indicate which energy parametrization is used in the calculation (the energy parametrization 1 (2) is described in section 3.1 (3.2), respectively), and, finally, the last column provides the name of the corresponding expression in the supplementary material.

with constant letters and argument $x_{1}$ following the procedure sometimes referred to as the "super-shuffle" [57]. Once this is done, the integration over $x_{1}$ becomes straightforward as well. We note that the final result for $\mathcal{I}_{\mathrm{TC}}$ is naturally expressed in terms of GPLs of weights up to four, with rational letters and arguments; to write them in terms of standard set of weight-four constants, we numerically evaluate GPLs using GINAC [58] and use PSLQ to fit them to linear combinations of relevant transcendental and rational numbers.

\section{$7 \quad$ Results for integrated triple-collinear subtraction terms}

Following the discussion in the previous sections, we calculated triple-collinear subtraction terms, for both initial and final state radiation, for all partonic channels that are required to compute NNLO QCD corrections to arbitrary hard processes at the LHC. We illustrate these results in this section. We note that all integrated triple-collinear subtraction terms presented in this section have been checked against the results of numerical integration. The complete set of results can be found in the supplementary material provided with this paper.

As a final remark we note that description of the initial state radiation requires that a crossing from final to initial states is performed in the splitting functions presented in ref. [49]. Moreover, for non-diagonal splittings $i \rightarrow j^{*}$ a different color-averaging is required when collinear limits of differential cross-sections are expressed in terms of the splitting functions. To avoid potential confusion related to these subtleties, in table 1 (2) we list the partonic channels for relevant initial (final) state splittings, the corresponding splitting functions in [49] and specific details related to the energy integrations. We also emphasize 


\begin{tabular}{|l|c|c|c|}
\hline Splitting & $P_{a b c}$ & $n$ & Name in the supplementary material \\
\hline$q^{*} \rightarrow g g q$ & $1 / 2\left(P_{g_{4} g_{5} q_{1}}+4 \leftrightarrow 5\right)$ & 1 & FSR $[1]$ \\
$q^{*} \rightarrow \bar{q}^{\prime} q^{\prime} q$ & $P_{\bar{q}_{4}^{\prime} q_{5}^{\prime} q_{1}}+4 \leftrightarrow 5$ & 1 & FSR $[2]$ \\
$q^{*} \rightarrow \bar{q} q q$ & $P_{\bar{q}_{4} q_{1} q_{5}}+4 \leftrightarrow 5$ & 1 & FSR $[3]$ \\
$g^{*} \rightarrow g q \bar{q}$ & $P_{g_{1} q_{4} \bar{q}_{5}}+P_{g_{4} q_{1} \bar{q}_{5}}+P_{g_{5} q_{1} \bar{q}_{4}}$ & 2 & FSR[4] \\
$g^{*} \rightarrow g g g$ & $P_{g_{1} g_{4} g_{5}}$ & 2 & FSR [5] \\
\hline
\end{tabular}

Table 2. Overview of integrated triple-collinear subtraction terms for final state splittings. In the first column we define the splitting, in the second column, we identify the corresponding triplecollinear splitting functions in ref. [49], where we include an additional symmetry factor where required. The fifth column denotes the power $n$ as defined in eq. (6.1) and the last column provides, again, the name of the corresponding expression in the supplementary material.

that we do not introduce new color averages factors into our formulas so that the color factors in our results are the same as in the splitting functions in ref. [49].

\subsection{Initial state radiation}

In this section we illustrate the results of the computation by providing integrated triplecollinear subtraction terms for two partonic channels, $q \rightarrow q^{*}+g g$ and $g \rightarrow q^{*}+q g$. We begin with the presentation of our results for the integrated triple-collinear splitting $q \rightarrow q^{*}+g g$ that corresponds to the splitting function $P_{g g q}$ in ref. [49]. Since this process exhibits a double-soft singularity, we use the parameterization of energies given in eq. (3.8) to integrate triple-collinear subtraction terms. To present the result, we decompose it into the color factors

$$
R_{\delta,+, \mathrm{reg}}=C_{F}^{2} R_{\delta,+, \mathrm{reg}}^{\mathrm{A}}+C_{F} C_{A} R_{\delta,+, \mathrm{reg}}^{\mathrm{NA}},
$$

and obtain

$$
\begin{aligned}
R_{\delta}^{\mathrm{A}}= & \frac{1}{\epsilon}\left(\frac{\pi^{2}}{3} \ln (2)\right)-\frac{7 \pi^{2}}{6} \ln ^{2}(2)+8 \zeta_{3} \ln (2) \\
R_{\delta}^{\mathrm{NA}}= & \frac{1}{\epsilon}\left(-\frac{1571}{216}+\frac{11 \pi^{2}}{36}+\frac{3}{8} \zeta_{3}+\frac{\pi^{2}}{3} \ln (2)+\frac{11}{2} \ln ^{2}(2)+\left(-\frac{32}{9}+\frac{\pi^{2}}{6}-\frac{11 \ln (2)}{3}\right) \ln \left(E_{\max } / E_{1}\right)\right) \\
& -\frac{1}{12} \ln ^{4}(2)-\frac{176}{9} \ln ^{3}(2)-\left(\frac{79}{9}+\frac{11 \pi^{2}}{12}\right) \ln ^{2}(2)+\frac{513 \zeta_{3}+913+165 \pi^{2}}{108} \ln (2) \\
& +\left(\frac{64}{9}-\frac{\pi^{2}}{3}+\frac{22 \ln (2)}{3}\right) \ln ^{2}\left(E_{\max } / E_{1}\right) \\
& +\left(\frac{11 \zeta_{3}}{2}+\frac{383}{54}-\frac{22 \pi^{2}}{9}-11 \ln ^{2}(2)+\frac{\ln (2)}{3}-\frac{2}{3} \pi^{2} \ln (2)\right) \ln \left(E_{\max } / E_{1}\right) \\
R_{+}^{A}= & -\frac{4 \pi^{2}}{3} \ln (2), \\
R_{+}^{\mathrm{NA}}= & \frac{1}{\epsilon}\left(\frac{11}{3} \ln (2)-\frac{\pi^{2}}{6}+\frac{32}{9}\right)-11 \ln ^{2}(2)-\frac{1+2 \pi^{2}}{3} \ln (2)-7 \zeta_{3}+\frac{11 \pi^{2}}{9}+22 .
\end{aligned}
$$


Results for the regular parts are more complex. We find

$$
\begin{aligned}
R_{\mathrm{reg}}^{\mathrm{A}}= & \frac{1}{\epsilon}\left(-\frac{z+1}{2} \ln (2) \ln (z)+(1-z) \ln (2)+\frac{\left(z^{2}+3\right)}{4(z-1)} \ln ^{2}(z)-\ln (z) z+\frac{3(z-1)}{2}\right) \\
& +\frac{z^{2}\left(-36 \zeta_{3}+33+4 \pi^{2}\right)-2\left(33+2 \pi^{2}\right) z-60 \zeta_{3}+33}{6(z-1)}+\frac{7(z-1)}{2} \ln ^{2}(2) \\
& +\left(-6 z+\pi^{2}(z+1)+6\right) \ln (2)+\frac{\left(3(z-1) z-\pi^{2}\left(3 z^{2}+5\right)\right)}{3(z-1)} \ln (z) \\
& +\frac{z}{2} \ln ^{2}(z)+\frac{\left(9 z^{2}+19\right)}{12(1-z)} \ln ^{3}(z)+\frac{7(z+1)}{4} \ln ^{2}(2) \ln (z)+\frac{\left(z^{2}+7\right)}{2(1-z)} \ln (2) \ln ^{2}(z) \\
& +(3 z-1) \ln (2) \ln (z)+6(1-z) \ln (1-z)-4(1-z) \ln (1-z) \ln (2) \\
& +\left(-2(z+1) \ln (2)-\frac{2\left(z^{2}+1\right)}{z-1} \ln (z)-4 z\right) \operatorname{Li}_{2}(z)+\left(\frac{2\left(3 z^{2}+5\right)}{z-1}\right) \operatorname{Li}_{3}(z),
\end{aligned}
$$

for the abelian part and

$$
\begin{aligned}
R_{\mathrm{reg}}^{\mathrm{NA}}= & \frac{1}{\epsilon}\left(\frac{\left(6 \pi^{2}-61\right) z^{2}-15 z+76}{36(z-1)}-\frac{11(z+1)}{6} \ln (2)+\frac{\left(11 z^{2}+2\right)}{12(z-1)} \ln (z)\right. \\
& \left.+\frac{\left(z^{2}+1\right)}{2(1-z)} \ln (1-z) \ln (z)+\left(\frac{1+z^{2}}{2(1-z)}\right) \operatorname{Li}_{2}(z)\right) \\
& +\frac{3\left(z^{2}\left(48 \zeta_{3}-119\right)-46 z-36 \zeta_{3}+165\right)+\pi^{2}\left(-50 z^{2}+12 z+12\right)}{36(z-1)} \\
& +\frac{\left(\left(61-6 \pi^{2}\right) z^{2}+15 z-76\right)}{9(z-1)} \ln (1-z)+\frac{\left(49 z^{2}+57 z-20\right)}{36(z-1)} \ln (z) \\
& +\frac{2\left(z^{2}+1\right)}{z-1} \ln ^{2}(1-z) \ln (z)+\frac{(z-1)}{2} \ln (1-z) \ln (z)+\frac{\left(11 z^{2}+2\right)}{8(1-z)} \ln 2(z) \\
& +\frac{2\left(z^{2}+1\right)}{z-1} \ln (1-z) \ln (z) \ln (2)+\frac{22(z+1)}{3} \ln (1-z) \ln (2)+\frac{\left(z^{2}+1\right)}{4(z-1)} \ln (1-z) \ln ^{2}(z) \\
& +\frac{11(z+1)}{2} \ln ^{2}(2)+\frac{\left(11 z^{2}+2\right)}{3(1-z)} \ln (2) \ln (z)+\frac{\left(-7 z^{2}+6 z+4 \pi^{2}+1\right)}{6(1-z)} \ln (2) \\
& +\left(\frac{2\left(z^{2}+1\right)}{z-1} \ln (1-z)+\frac{2\left(z^{2}+1\right)}{z-1} \ln (2)+\frac{\left(z^{2}+1\right)}{2(z-1)} \ln (z)+\frac{25 z^{2}-6 z+7}{6(z-1)}\right) \operatorname{Li}_{2}(z) \\
& +\left(\frac{2\left(z^{2}+1\right)}{z-1}\right) \operatorname{Li} 3(1-z)+\left(\frac{\left(z^{2}+1\right)}{2(1-z)}\right) \operatorname{Li}_{3}(z),
\end{aligned}
$$

for the non-abelian. As the second example, we present the results for the integrated triple-collinear counter-term that describes the $g \rightarrow q^{*}+q g$ splitting. Since in this case there is no double-soft singularity, we use the energy parametrization given in eq. (3.17). 
Another consequence of the absence of the double-soft singularity is the regularity of the counter-term so that it does not contain either a $\delta(1-z)$ function or a plus-distribution. We decompose the result into the color factors

$$
\tilde{R}_{\mathrm{reg}}=C_{F}^{2} \tilde{R}_{\mathrm{reg}}^{\mathrm{A}}+C_{F} C_{A} \tilde{R}_{\mathrm{reg}}^{\mathrm{NA}}
$$

and find

$$
\begin{aligned}
& \tilde{R}_{\mathrm{reg}}^{\mathrm{A}}=\frac{1}{\epsilon}\left(\frac{8 \pi^{2} z^{2}-8 \pi^{2} z-15 z+4 \pi^{2}-3}{12}+3\left(2 z^{2}-2 z+1\right) \ln (1-z) \ln (2)\right. \\
& +\left(-2 z^{2}+2 z-1\right) \ln (1-z) \ln (z)+\frac{1-2 z}{2} \ln (z) \ln (2)+\frac{-9 z^{2}+11 z-5}{2} \ln (2) \\
& +\frac{4 z^{2}-6 z+3}{4} \ln ^{2}(z)-\frac{3}{4} \ln (z)-\left(2 z^{2}-2 z+1\right) \operatorname{Li}_{2}(z) \\
& \left.-3\left(1-2 z+2 z^{2}\right) \ln (2) \ln \left(E_{\max } / E_{1}\right)\right) \\
& +\frac{-3 \pi^{2} z^{2}+12 z \zeta_{3}+3 \pi^{2} z-24 z-6 \zeta_{3}-\pi^{2}}{3}-9\left(2 z^{2}-2 z+1\right) \ln ^{2}(1-z) \ln (2) \\
& +4\left(2 z^{2}-2 z+1\right) \ln ^{2}(1-z) \ln (z)-\frac{19\left(2 z^{2}-2 z+1\right)}{2} \ln (1-z) \ln ^{2}(2) \\
& +4\left(2 z^{2}-2 z+1\right) \ln (1-z) \ln (2) \ln (2)+\left(18 z^{2}-22 z+7\right) \ln (1-z) \ln (2) \\
& +\frac{\left(2 z^{2}-2 z+1\right)}{2} \ln (1-z) \ln ^{2}(z)+\ln (1-z) \ln (z)+\frac{7(2 z-1)}{4} \ln (z) \ln ^{2}(2) \\
& +\frac{3-4 \pi^{2} z^{2}+4 \pi^{2} z+15 z-2 \pi^{2}}{3} \ln (1-z)+\frac{57 z^{2}-71 z+32}{4} \ln ^{2}(2) \\
& +\frac{-8 z^{2}+14 z-7}{2} \ln ^{2}(z) \ln (2)+2(z+2) \ln (z) \ln (2) \\
& +\frac{-4 \pi^{2} z^{2}-117 z^{2}+8 \pi^{2} z+150 z-4 \pi^{2}-27}{6} \ln (2)+\frac{-28 z^{2}+38 z-19}{12} \ln ^{3}(z) \\
& +\frac{(8 z+9)}{8} \ln ^{2}(z)+\frac{-32 \pi^{2} z^{2}+40 \pi^{2} z-21 z-20 \pi^{2}+9}{12} \ln (z) \\
& +\left(\ln (2)\left(8 z^{2}-12 z+6\right)+\left(-2 z^{2}+2 z-1\right)(\ln (z)-4 \ln (1-z))-2\right) \operatorname{Li}_{2}(z) \\
& +\left(8 z^{2}-8 z+4\right) \operatorname{Li}_{3}(1-z)+\left(14 z^{2}-18 z+9\right) \operatorname{Li}_{3}(z) \\
& +3\left(1-2 z+2 z^{2}\right) \ln (2) \ln ^{2}\left(E_{\max } / E_{1}\right) \\
& +\left(\frac{19\left(1-2 z+2 z^{2}\right)}{2} \ln ^{2}(2)+6\left(1-2 z+2 z^{2}\right) \ln (1-z) \ln (2)+3 \ln (2)\right. \\
& \left.-\frac{2 \pi^{2}\left(1-2 z+2 z^{2}\right)}{3}\right) \ln \left(E_{\max } / E_{1}\right),
\end{aligned}
$$


and

$$
\begin{aligned}
\tilde{R}_{\mathrm{reg}}^{\mathrm{NA}}= & \frac{1}{\epsilon}\left(\frac{-6 \pi^{2} z^{3}-67 z^{3}+3 \pi^{2} z^{2}+81 z^{2}-3 \pi^{2} z-27 z+13}{9 z}\right. \\
& +\left(2 z^{2}-2 z+1\right) \ln (1-z) \ln (2)+\left(2 z^{2}-2 z+1\right) \ln (1-z) \ln (z) \\
& -\left(2 z^{2}+2 z+1\right) \ln (1+z) \ln (z)+(4 z+1) \ln (z) \ln (2)+\frac{4-31 z^{3}+24 z^{2}+3 z}{6 z} \ln (2) \\
& +\frac{6 z+1}{2} \ln ^{2}(z)+\frac{12 z+1}{2} \ln (z)-\left(2 z^{2}+2 z+1\right) \operatorname{Li}_{2}(-z)+\left(2 z^{2}-2 z+1\right) \operatorname{Li}_{2}(z) \\
& \left.-\left(1-2 z+2 z^{2}\right) \ln (2) \ln \left(E_{\max } / E_{1}\right)\right) \\
& +\left(\left(8 z^{2}+8 z+4\right)(\ln (1-z)+\ln (2))+\left(2 z^{2}-6 z+1\right) \ln (z)\right) \operatorname{Li}_{2}(-z) \\
& +\left(\left(-8 z^{2}+8 z-4\right) \ln (1-z)-8(z-3) z \ln (2)-4 z \ln (z)\right) \operatorname{Li}_{2}(z) \\
& +\frac{44 z^{3}+48 z^{2}+15 z+8}{3 z} \operatorname{Li}_{2}(-z)+\frac{-22 z^{3}+96 z^{2}-3 z+20}{3 z} \operatorname{Li}_{2}(z) \\
& -\left(18 z^{2}-2 z+9\right) \operatorname{Li}_{3}(1-z)+\left(10 z^{2}+26 z+5\right) \operatorname{Li}_{3}(-z) \\
& +\left(4 z^{2}+4 z+2\right)\left(3 \operatorname{Li}_{3}\left(\frac{z}{1+z}\right)+\operatorname{Li}_{3}\left(1-z^{2}\right)\right)+(32 z+4) \operatorname{Li}_{3}(z) \\
& +\left(1-2 z+2 z^{2}\right) \ln (2) \ln { }^{2}\left(E_{\max } / E_{1}\right) \\
& +\left(\frac{7\left(1-2 z+2 z^{2}\right)}{2} \ln (2)+2\left(1-2 z+2 z^{2}\right) \ln (1-z)+1\right) \ln (2) \ln \left(E_{\max } / E_{1}\right) .
\end{aligned}
$$

Results for other integrated triple-collinear counter-terms are of a similar complexity. They can be found in an supplementary material provided with this paper.

\subsection{Final state radiation}

In this section we present the results for the integrated triple-collinear counter-terms relevant for two partonic channels, $q^{*} \rightarrow g g q$ and $q^{*} \rightarrow \bar{q} q^{\prime} \bar{q}^{\prime}$. Following ref. [49], we split the three-quark triple-collinear splitting function into two contributions

$$
P_{\bar{q}_{1} q_{2} q_{3}}=P_{\bar{q}_{1}^{\prime} q_{2}^{\prime} q_{3}}+P_{\bar{q}_{1} q_{2} q_{3}}^{\mathrm{id}},
$$

that allow a description of final states with both identical and different quark flavors. We present the corresponding contributions separately.

To present the results, we define

$$
\mathcal{I}_{\mathrm{TC}}^{q a b}=\left[\alpha_{s}\right]^{2} E^{-4 \epsilon} R^{q a b},
$$


and obtain

$$
\begin{aligned}
R^{q g g}= & C_{A} C_{F}\left\{\frac{1}{\epsilon}\left[-\frac{1015}{108}+\frac{19 \zeta_{3}}{8}+\frac{\pi^{2}}{8}+\frac{11}{2} \ln ^{2}(2)-\frac{11}{4} \ln (2)+\frac{1}{3} \pi^{2} \ln (2)\right]\right. \\
& +\left[-\frac{2281}{48}-2 \operatorname{Li}_{4}(1 / 2)+\frac{25 \zeta_{3}}{24}-\frac{13}{4} \zeta_{3} \ln (2)-\frac{119 \pi^{2}}{144}+\frac{173 \pi^{4}}{480}-\frac{\ln ^{4}(2)}{12}\right. \\
& \left.\left.-\frac{176}{9} \ln ^{3}(2)-\frac{19}{36} \ln ^{2}(2)-\frac{11}{12} \pi^{2} \ln ^{2}(2)-\frac{1247}{108} \ln (2)+\frac{161}{36} \pi^{2} \ln (2)\right]\right\} \\
& +C_{F}^{2}\left\{\frac{1}{\epsilon}\left[\frac{31}{16}-2 \zeta_{3}+\frac{9}{8} \ln (2)+\frac{1}{3} \pi^{2} \ln (2)\right]+\left[\frac{715}{32}+16 \zeta_{3} \ln (2)-\frac{7 \pi^{4}}{30}\right.\right. \\
& \left.\left.-\frac{63}{16} \ln ^{2}(2)-\frac{7}{6} \pi^{2} \ln ^{2}(2)+\frac{17}{8} \ln (2)+\pi^{2} \ln (2)\right]\right\} \\
R^{q q^{\prime} q^{\prime}}= & C_{F} T_{R}\left\{\frac{1}{\epsilon}\left[\frac{329}{108}-2 \ln { }^{2}(2)+\ln (2)\right]+\left[\frac{2773}{216}+\frac{19 \zeta_{3}}{6}\right.\right. \\
& \left.\left.+\frac{35 \pi^{2}}{72}+\frac{64}{9} \ln ^{3}(2)+\frac{32}{9} \ln ^{2}(2)+\frac{43}{27} \ln (2)-\frac{13}{9} \pi^{2} \ln (2)\right]\right\} \\
R^{q \bar{q} q, \text { id }}= & C_{F}\left(C_{F}-\frac{1}{2} C_{A}\right)\left\{\frac{1}{\epsilon}\left[-\frac{13}{4}-2 \zeta_{3}+\frac{\pi^{2}}{2}\right]\right. \\
& \left.+\left[-\frac{335}{8}+39 \zeta_{3}+8 \zeta_{3} \ln (2)+\frac{5 \pi^{2}}{3}-\frac{14 \pi^{4}}{45}+13 \ln (2)-2 \pi^{2} \ln (2)\right]\right\}
\end{aligned}
$$

\section{Conclusion}

In this paper, we computed all the relevant integrated triple-collinear subtraction terms for both initial and final state radiation in the context of the nested soft-collinear subtraction scheme of ref. [1]. Together with the results for the integrated double-soft eikonal factor presented earlier in ref. [47], the computation reported in this paper completes the calculation of the required integrated subtraction terms for the subtraction scheme of ref. [1]. In addition to improving the efficiency and numerical stability of practical computations, these results should enable the derivation of a general NNLO QCD subtraction formula for arbitrary hard processes at the LHC, similar to Catani-Seymour [59] and FKS [60, 61] schemes at NLO QCD.

\section{Acknowledgments}

We are grateful to F. Caola and R. Roentsch for useful conversations. We would like to thank Ch. Wever for his help with Mellin-Barnes integration. The research of K.M. is supported by BMBF grant 05H18VKCC1 and by the DFG Collaborative Research Center TRR 257 "Particle Physics Phenomenology after the Higgs Discovery". M.D. is supported by the start-up funds provided by Karlsruhe Institute of Technology. 
Open Access. This article is distributed under the terms of the Creative Commons Attribution License (CC-BY 4.0), which permits any use, distribution and reproduction in any medium, provided the original author(s) and source are credited.

\section{References}

[1] F. Caola, K. Melnikov and R. Röntsch, Nested soft-collinear subtractions in NNLO QCD computations, Eur. Phys. J. C 77 (2017) 248 [arXiv: 1702.01352] [INSPIRE].

[2] L. Chen, M. Czakon and R. Poncelet, Polarized double-virtual amplitudes for heavy-quark pair production, JHEP 03 (2018) 085 [arXiv: 1712.08075] [INSPIRE].

[3] T. Gehrmann, A. von Manteuffel and L. Tancredi, The two-loop helicity amplitudes for $q \bar{q}^{\prime} \rightarrow V_{1} V_{2} \rightarrow 4$ leptons, JHEP 09 (2015) 128 [arXiv: 1503.04812] [INSPIRE].

[4] F. Caola, J.M. Henn, K. Melnikov, A.V. Smirnov and V.A. Smirnov, Two-loop helicity amplitudes for the production of two off-shell electroweak bosons in quark-antiquark collisions, JHEP 11 (2014) 041 [arXiv: 1408.6409] [INSPIRE].

[5] T. Gehrmann, M. Jaquier, E.W.N. Glover and A. Koukoutsakis, Two-Loop QCD Corrections to the Helicity Amplitudes for $H \rightarrow 3$ partons, JHEP 02 (2012) 056 [arXiv:1112.3554] [INSPIRE].

[6] L.W. Garland, T. Gehrmann, E.W.N. Glover, A. Koukoutsakis and E. Remiddi, The Two loop QCD matrix element for $e^{+} e^{-} \rightarrow 3$ jets, Nucl. Phys. B 627 (2002) 107 [hep-ph/0112081] [INSPIRE].

[7] C. Anastasiou, E.W.N. Glover, C. Oleari and M.E. Tejeda-Yeomans, Two-loop QCD corrections to the scattering of massless distinct quarks, Nucl. Phys. B 601 (2001) 318 [hep-ph/0010212] [INSPIRE].

[8] A. Gehrmann-De Ridder, T. Gehrmann and E.W.N. Glover, Antenna subtraction at NNLO, JHEP 09 (2005) 056 [hep-ph/0505111] [INSPIRE].

[9] A. Gehrmann-De Ridder, T. Gehrmann and E.W.N. Glover, Gluon-gluon antenna functions from Higgs boson decay, Phys. Lett. B 612 (2005) 49 [hep-ph/0502110] [INSPIRE].

[10] A. Gehrmann-De Ridder, T. Gehrmann and E.W.N. Glover, Quark-gluon antenna functions from neutralino decay, Phys. Lett. B 612 (2005) 36 [hep-ph/0501291] [INSPIRE].

[11] A. Daleo, T. Gehrmann and D. Maître, Antenna subtraction with hadronic initial states, JHEP 04 (2007) 016 [hep-ph/0612257] [INSPIRE].

[12] A. Daleo, A. Gehrmann-De Ridder, T. Gehrmann and G. Luisoni, Antenna subtraction at NNLO with hadronic initial states: initial-final configurations, JHEP 01 (2010) 118 [arXiv: 0912.0374] [INSPIRE].

[13] T. Gehrmann and P.F. Monni, Antenna subtraction at NNLO with hadronic initial states: real-virtual initial-initial configurations, JHEP 12 (2011) 049 [arXiv:1107.4037] [INSPIRE].

[14] R. Boughezal, A. Gehrmann-De Ridder and M. Ritzmann, Antenna subtraction at NNLO with hadronic initial states: double real radiation for initial-initial configurations with two quark flavours, JHEP 02 (2011) 098 [arXiv: 1011.6631] [INSPIRE].

[15] A. Gehrmann-De Ridder, T. Gehrmann and M. Ritzmann, Antenna subtraction at NNLO with hadronic initial states: double real initial-initial configurations, JHEP 10 (2012) 047 [arXiv: 1207.5779] [INSPIRE]. 
[16] J. Currie, E.W.N. Glover and S. Wells, Infrared Structure at NNLO Using Antenna Subtraction, JHEP 04 (2013) 066 [arXiv: 1301.4693] [INSPIRE].

[17] R. Boughezal, C. Focke, X. Liu and F. Petriello, $W$-boson production in association with a jet at next-to-next-to-leading order in perturbative QCD, Phys. Rev. Lett. 115 (2015) 062002 [arXiv: 1504.02131] [INSPIRE].

[18] J. Gaunt, M. Stahlhofen, F.J. Tackmann and J.R. Walsh, N-jettiness Subtractions for NNLO QCD Calculations, JHEP 09 (2015) 058 [arXiv: 1505.04794] [INSPIRE].

[19] S. Catani and M. Grazzini, An NNLO subtraction formalism in hadron collisions and its application to Higgs boson production at the LHC, Phys. Rev. Lett. 98 (2007) 222002 [hep-ph/0703012] [INSPIRE].

[20] M. Grazzini, NNLO predictions for the Higgs boson signal in the $H \rightarrow W W \rightarrow l \nu l \nu$ and $H \rightarrow Z Z \rightarrow 4 l$ decay channels, JHEP 02 (2008) 043 [arXiv:0801.3232] [InSPIRE].

[21] M. Czakon, A novel subtraction scheme for double-real radiation at NNLO, Phys. Lett. B 693 (2010) 259 [arXiv:1005.0274] [INSPIRE].

[22] M. Czakon, Double-real radiation in hadronic top quark pair production as a proof of a certain concept, Nucl. Phys. B 849 (2011) 250 [arXiv:1101.0642] [INSPIRE].

[23] M. Czakon and D. Heymes, Four-dimensional formulation of the sector-improved residue subtraction scheme, Nucl. Phys. B 890 (2014) 152 [arXiv:1408.2500] [INSPIRE].

[24] F. Herzog, Geometric IR subtraction for final state real radiation, JHEP 08 (2018) 006 [arXiv: 1804.07949] [INSPIRE].

[25] L. Magnea, E. Maina, G. Pelliccioli, C. Signorile-Signorile, P. Torrielli and S. Uccirati, Local Analytic Sector Subtraction at NNLO, JHEP 12 (2018) 107 [arXiv: 1806.09570] [INSPIRE].

[26] M. Czakon, P. Fiedler and A. Mitov, Total Top-Quark Pair-Production Cross Section at Hadron Colliders Through $\mathcal{O}\left(\alpha_{S}^{4}\right)$, Phys. Rev. Lett. 110 (2013) 252004 [arXiv:1303.6254] [INSPIRE].

[27] M. Czakon, P. Fiedler and A. Mitov, Resolving the Tevatron Top Quark Forward-Backward Asymmetry Puzzle: Fully Differential Next-to-Next-to-Leading-Order Calculation, Phys. Rev. Lett. 115 (2015) 052001 [arXiv:1411.3007] [INSPIRE].

[28] M. Czakon, D. Heymes and A. Mitov, High-precision differential predictions for top-quark pairs at the LHC, Phys. Rev. Lett. 116 (2016) 082003 [arXiv:1511.00549] [INSPIRE].

[29] M. Czakon, P. Fiedler, D. Heymes and A. Mitov, NNLO QCD predictions for fully-differential top-quark pair production at the Tevatron, JHEP 05 (2016) 034 [arXiv: 1601.05375] [INSPIRE].

[30] M. Brucherseifer, F. Caola and K. Melnikov, On the NNLO QCD corrections to single-top production at the LHC, Phys. Lett. B 736 (2014) 58 [arXiv:1404.7116] [InSPIRE].

[31] E.L. Berger, J. Gao, C.P. Yuan and H.X. Zhu, NNLO QCD Corrections to t-channel Single Top-Quark Production and Decay, Phys. Rev. D 94 (2016) 071501 [arXiv:1606.08463] [INSPIRE].

[32] X. Chen, T. Gehrmann, E.W.N. Glover and M. Jaquier, Precise QCD predictions for the production of Higgs + jet final states, Phys. Lett. B 740 (2015) 147 [arXiv:1408.5325] [INSPIRE]. 
[33] R. Boughezal, F. Caola, K. Melnikov, F. Petriello and M. Schulze, Higgs boson production in association with a jet at next-to-next-to-leading order, Phys. Rev. Lett. 115 (2015) 082003 [arXiv: 1504.07922] [INSPIRE].

[34] F. Caola, K. Melnikov and M. Schulze, Fiducial cross sections for Higgs boson production in association with a jet at next-to-next-to-leading order in $Q C D$, Phys. Rev. D 92 (2015) 074032 [arXiv: 1508.02684] [INSPIRE].

[35] X. Chen, J. Cruz-Martinez, T. Gehrmann, E.W.N. Glover and M. Jaquier, NNLO QCD corrections to Higgs boson production at large transverse momentum, JHEP 10 (2016) 066 [arXiv: 1607.08817] [INSPIRE].

[36] R. Boughezal, C. Focke, W. Giele, X. Liu and F. Petriello, Higgs boson production in association with a jet at NNLO using jettiness subtraction, Phys. Lett. B 748 (2015) 5 [arXiv: 1505.03893] [INSPIRE].

[37] R. Boughezal, X. Liu and F. Petriello, W-boson plus jet differential distributions at NNLO in QCD, Phys. Rev. D 94 (2016) 113009 [arXiv:1602.06965] [INSPIRE].

[38] A. Gehrmann-De Ridder, T. Gehrmann, E.W.N. Glover, A. Huss and T.A. Morgan, Precise $Q C D$ predictions for the production of a $Z$ boson in association with a hadronic jet, Phys. Rev. Lett. 117 (2016) 022001 [arXiv:1507.02850] [INSPIRE].

[39] A. Gehrmann-De Ridder, T. Gehrmann, E.W.N. Glover, A. Huss and T.A. Morgan, The $N N L O Q C D$ corrections to $Z$ boson production at large transverse momentum, JHEP 07 (2016) 133 [arXiv: 1605.04295] [INSPIRE].

[40] A. Gehrmann-De Ridder, T. Gehrmann, E.W.N. Glover, A. Huss and T.A. Morgan, NNLO

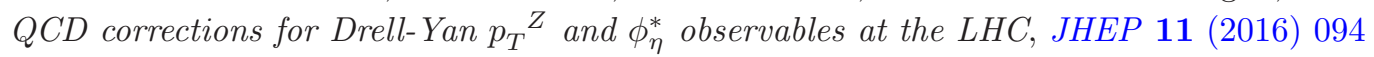
[Erratum JHEP 10 (2018) 126] [arXiv:1610.01843] [INSPIRE].

[41] R. Boughezal et al., Z-boson production in association with a jet at next-to-next-to-leading order in perturbative QCD, Phys. Rev. Lett. 116 (2016) 152001 [arXiv:1512.01291] [INSPIRE].

[42] R. Boughezal, X. Liu and F. Petriello, A comparison of NNLO QCD predictions with $7 \mathrm{TeV}$ ATLAS and CMS data for $V+$ jet processes, Phys. Lett. B 760 (2016) 6 [arXiv: 1602.05612] [INSPIRE].

[43] R. Boughezal, X. Liu and F. Petriello, Phenomenology of the Z-boson plus jet process at NNLO, Phys. Rev. D 94 (2016) 074015 [arXiv: 1602.08140] [InSPIRE].

[44] J. Currie, A. Gehrmann-De Ridder, T. Gehrmann, E.W.N. Glover, A. Huss and J. Pires, Precise predictions for dijet production at the LHC, Phys. Rev. Lett. 119 (2017) 152001 [arXiv: 1705.10271] [INSPIRE].

[45] M. Cacciari, F.A. Dreyer, A. Karlberg, G.P. Salam and G. Zanderighi, Fully Differential Vector-Boson-Fusion Higgs Production at Next-to-Next-to-Leading Order, Phys. Rev. Lett. 115 (2015) 082002 [Erratum ibid. 120 (2018) 139901] [arXiv:1506. 02660] [INSPIRE].

[46] J. Cruz-Martinez, T. Gehrmann, E.W.N. Glover and A. Huss, Second-order QCD effects in Higgs boson production through vector boson fusion, Phys. Lett. B 781 (2018) 672 [arXiv: 1802.02445] [INSPIRE].

[47] F. Caola, M. Delto, H. Frellesvig and K. Melnikov, The double-soft integral for an arbitrary angle between hard radiators, Eur. Phys. J. C 78 (2018) 687 [arXiv:1807.05835] [INSPIRE]. 
[48] C. Anastasiou and K. Melnikov, Higgs boson production at hadron colliders in NNLO QCD, Nucl. Phys. B 646 (2002) 220 [hep-ph/0207004] [INSPIRE].

[49] S. Catani and M. Grazzini, Infrared factorization of tree level QCD amplitudes at the next-to-next-to-leading order and beyond, Nucl. Phys. B 570 (2000) 287 [hep-ph/9908523] [INSPIRE].

[50] F. Caola, K. Melnikov and R. Roentsch, Analytic results for color-singlet production at NNLO QCD with the nested soft-collinear subtraction scheme, arXiv:1902.02081 [INSPIRE].

[51] A. von Manteuffel and C. Studerus, Reduze 2 - Distributed Feynman Integral Reduction, arXiv: 1201.4330 [INSPIRE].

[52] J.M. Henn, Multiloop integrals in dimensional regularization made simple, Phys. Rev. Lett. 110 (2013) 251601 [arXiv:1304.1806] [INSPIRE].

[53] R.N. Lee, Reducing differential equations for multiloop master integrals, JHEP 04 (2015) 108 [arXiv: 1411.0911] [INSPIRE].

[54] A.B. Goncharov, Polylogarithms in Arithmetic and Geometry, in proceedings of the International Congress of Mathematicians, Zürich, Switzerland, 3-11 August 1994, Birkhäuser, Basel Switzerland, pp. 374-387.

[55] M. Czakon, Automatized analytic continuation of Mellin-Barnes integrals, Comput. Phys. Commun. 175 (2006) 559 [hep-ph/0511200] [INSPIRE].

[56] F. Caola, G. Luisoni, K. Melnikov and R. Röntsch, NNLO QCD corrections to associated $W H$ production and $H \rightarrow b \bar{b}$ decay, Phys. Rev. D 97 (2018) 074022 [arXiv:1712.06954] [INSPIRE].

[57] H. Frellesvig, Generalized Polylogarithms in Maple, arXiv:1806.02883 [INSPIRE].

[58] J. Vollinga and S. Weinzierl, Numerical evaluation of multiple polylogarithms, Comput. Phys. Commun. 167 (2005) 177 [hep-ph/0410259] [INSPIRE].

[59] S. Catani and M.H. Seymour, A General algorithm for calculating jet cross-sections in NLO QCD, Nucl. Phys. B 485 (1997) 291 [Erratum ibid. B 510 (1998) 503] [hep-ph/9605323] [INSPIRE].

[60] S. Frixione, Z. Kunszt and A. Signer, Three jet cross-sections to next-to-leading order, Nucl. Phys. B 467 (1996) 399 [hep-ph/9512328] [INSPIRE].

[61] S. Frixione, A General approach to jet cross-sections in QCD, Nucl. Phys. B 507 (1997) 295 [hep-ph/9706545] [INSPIRE]. 